\title{
Use of a blue filter in visual field analysis
}

\author{
Charles E Hugkulstone, Stephen A Vernon
}

\begin{abstract}
One hundred and twenty seven eyes of 127 patients were studied by means of a Kodak 47B Wratten blue gelatin filter in conjunction with the Friedmann Mark 1 visual field analyser. There were 49 patients in the normal group, 50 patients in the ocular hypertension group, and 28 patients in a group with early glaucoma. A simple scoring system did not show a useful difference between these three groups. However, use of a derived threshold value for each target group designated by the letters $B$ to $P$ (giving selective blue field scores) differentiated between normal subjects and those with early glaucomatous field loss. When used with a calculated upper limit of normal to give a specificity of $96 \%$, this test had a sensitivity of $64 \%$. Of the ocular hypertension group $22 \%$ gave abnormal results in comparison with normal persons. This simple adaptation to the Friedmann protocol may aid the ophthalmologist in the detection and confirmation of early glaucoma.
\end{abstract}

The diagnosis of chronic glaucoma generally rests on the triad of raised intraocular pressure, cupping of the optic disc, and a classical field defect. The diagnosis is straightforward when all three are present, but is less so in the early stages before unequivocal field defects develop.

Recent work has attempted to improve the sensitivity of detecting glaucomatous damage at an early stage. Blue light information is processed by larger sized ganglion cells (with corresponding differences in their axons) than red or green light, ${ }^{1}$ and it has been found that the larger diameter axons are selectively damaged by glaucoma. ${ }^{2}$ Blue colour vision defects have been shown to occur in glaucoma and ocular hypertension $^{3}$ and also to be related to the highest recorded intraocular pressure in patients with glaucoma. ${ }^{4}$ However, these tests require sophisticated and expensive equipment.

Only one study has investigated the use of a blue stimulus with a standard central field analyser, ${ }^{5}$ but it found no advantage for it over a white stimulus, though normal subjects were not studied. This paper presents the findings of a study by a different technique using a blue filter with a Friedmann central visual field analyser in normal subjects, ocular hypertensives, and patients with early chronic simple glaucoma.

\section{Patients and methods}

Subjects were selected from patients attending two separate ophthalmic outpatient departments who had a visual acuity of at least $6 / 18$ unaided and $6 / 9$ or better aided. Classification into the three groups (normal, ocular hypertension, or glaucoma) was based on the worse eye (that is, by patient). If both eyes were eligible for this study, then only one was randomly included in the statistical analysis.

Normal subjects were required to have an intraocular pressure of $21 \mathrm{mmHg}$ or less and healthy fundi with normal optic discs. Patients with glaucoma were defined by an intraocular pressure $>21 \mathrm{mmHg}$ without treatment, pathological cupping of the optic disc, and at least three adjacent spots of reduced sensitivity on field testing with the Friedmann Mark 1 analyser. However, if an eye had more than four points of absolute field loss per vertical hemifield, that eye was excluded from analysis, because we were concentrating on cases of early glaucoma only. The ocular hypertension group included patients with a similarly raised intraocular pressure but without visual field loss to a white target or specific pathological features on disc examination. Patients using miotic eye drops were excluded, as were known diabetics.

All patients gave verbal consent after the nature and purpose of this investigation had been explained to them.

The test was performed with the Friedmann Mark 1 visual field analyser without any spectacle correction. The threshold value for a white stimulus was determined by means of the four points $2.5^{\circ}$ from fixation (target group $P$ ). The neutral density filter was started at $2 \cdot 4 \log$ units, and subjects were allowed three attempts at this value. This was then reduced by steps of $0.2 \mathrm{log}$ unit until at least two of the four targets were seen in two out of the three attempts. This value was defined as the threshold, which was then reduced by a further $0.4 \log$ unit to run the test (the working value). The four extreme peripheral points (target group A) were ignored, and subjects were allowed three attempts at the working value for the remaining targets. If any points were missed, the filter setting was reduced by 0.2 $\log$ unit and a further three attempts were allowed at the new setting. This process was repeated until all the points were seen, and the highest filter setting for each point was recorded on a standard chart.

Once the test was completed for the white stimulus, a Kodak 47B Wratten blue gelatin filter mounted in a spectacle frame (with an occluder set in front of the fellow eye) was worn by the subject. We then repeated the test using the same protocol as above, but, unlike the previous report of this technique, ${ }^{5}$ we used the working value already determined for the white stimulus. A separate standard chart was used to record the highest filter setting found for each point with the blue stimulus.

Initially a simple scoring system was applied to the blue field. This gave 1 point for each $0.2 \mathrm{log}$ unit decrease from the working value found for all targets.

A further scoring system was also employed, 
Table 1 Mean (SD) values for blue field and selective blue field $(S B F)$ scores

\begin{tabular}{llc}
\hline & Blue field score & $S B F$ score \\
\hline Normal & $22 \cdot 3(25 \cdot 6)$ & $7 \cdot 5(6 \cdot 2)$ \\
Ocular hypertension & $31 \cdot 8(30 \cdot 7)$ & $12 \cdot 7(10 \cdot 4)$ \\
Glaucoma & $55 \cdot 2(31 \cdot 8)$ & $28 \cdot 7(15 \cdot 8)$ \\
\hline
\end{tabular}

termed the selective blue field (SBF). The SBF was obtained by considering the filter settings used for each one of the 14 groups of two to four individual targets when recording the blue field. The highest value recorded for any one of these points was treated as the threshold value for that particular target group. The remaining points in the group were then given a score of 1 point for each $0.2 \log$ unit reduction from this derived 'setting threshold'. These were then recorded on to a third standard chart, and their total score noted.

Statistical analysis was performed with the unpaired $t$ test. Values are given as mean (standard deviation) unless otherwise noted.

\section{Results}

In total 127 patients were studied. These were divided into 49 patients in the normal group, 50 in the ocular hypertension group, and 28 in the early glaucoma group. A further two patients were unable to see the fixation target when wearing the blue filter and were excluded from the study. The mean ages of these three groups, $58.7(10.2), 62.0(11.7)$, and $63.4(9.8)$ years respectively, showed no significant difference.

The mean blue field scores are shown in Table 1. Although there was a significant difference between the normal and glaucoma subjects, there was considerable overlap between all three groups because of the frequent generalised depression in sensitivity produced by the blue filter, especially within the central $10^{\circ}$. This is demonstrated by calculating an upper limit of normal as the mean $+(1.96 \times$ SD) from the results of the normal group. This gives a value of 72.5 , which is greater than the mean scores for both the ocular hypertension $(31.8)$ and the glaucoma groups $(55 \cdot 2)$. The blue field scores were therefore unhelpful in distinguishing patients with early glaucoma from normal subjects.

\section{SBF SCORE}

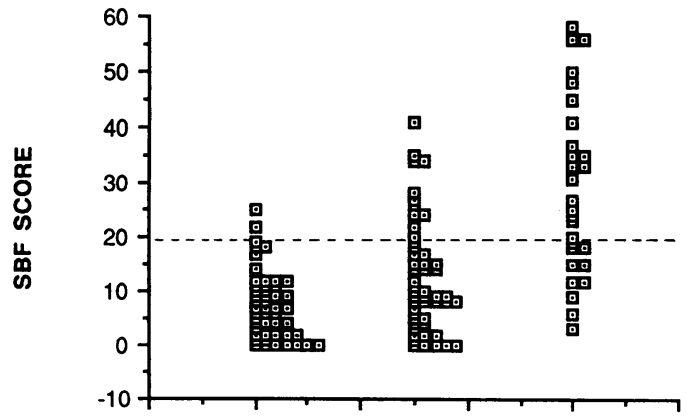

NORMAL

$\mathrm{OH}$

GLAUCOMA

Figure 1 Scattergram of the selective blue field $(S B F)$ scores for the three groups. Each point represents one eye of one patient. The dotted line represents the calculated upper limit of normal (see text). $\mathrm{OH}=$ ocular hypertension.
The SBF scoring system was developed to try to remove this background noise. Table 1 also shows the mean SBF scores for all three groups. The score for normal subjects was significantly lower than for both the ocular hypertension group $(p<0.01)$ and the glaucoma patients $(p<0.001)$, while the ocular hypertensive patients also had a lower SBF score than those with glaucoma $(p<0.001)$. The distribution of SBF scores in the three groups is presented in Figure 1.

An upper limit of normal of $19 \cdot 7$ for the SBF score was calculated in the same way as for the blue field score mentioned above. When this was applied to the ocular hypertensive group, although the mean score $(12 \cdot 7)$ was below the upper limit of normal, 11 subjects $(22 \%)$ had values above it. In the early glaucoma group 18 patients $(64 \%)$ had scores higher than the upper limit of normal, as was the mean score for this group (28.7).

The 10 eyes from the early glaucoma group that fall below the upper limit of normal were looked at more closely. Two had no field loss on standard testing with a white stimulus, but came from the patients whose diagnostic eye showed marked field loss and so was excluded from the analysis.

\section{Discussion}

The selection of subjects with good visual acuity was determined by the use of a spectacle frame to mount the blue filter. To prevent any artefacts arising from superimposing the blue filter frame over the patient's own spectacles we decided to test all subjects without any correction. By our choice of a minimum unaided acuity of $6 / 18$, we excluded any large uncorrected refractive error which might have influenced the results. Low power spherical refractive errors have been shown to reduce threshold values, but this occurs with a similar magnitude at all eccentricities within the central $25^{\circ},{ }^{6}$ and so should not affect our findings. However, it does mean that our results are confined to a select population, and further work would be required on patients whose visual acuities fall outside this range.

Although of interest, our original analyses using the blue field scores were clearly unhelpful in quantitatively distinguishing the glaucoma and normal groups. As mentioned above, this was because of the generalised depression in sensitivity with the blue field, particularly centrally, which the SBF was developed to counteract. We have demonstrated that the SBF score produces a marked difference between the normal and early glaucoma groups, with considerably less overlap.

In addition, when the specificity of the upper limit of normal for both SBF and blue field scores is set high at $96 \%$, the $\mathrm{SBF}$ score sensitivity for patients with early glaucoma is $64 \%$, in contrast to $25 \%$ for the blue field score. These values may reflect the relatively small numbers in the glaucoma group (all of whom had only early field changes), as the $95 \%$ confidence interval shows that the SBF sensitivity may range from $46 \%$ up to $82 \%$. If the specificity is lowered to $90 \%$, then the SBF sensitivity shows a 
corresponding increase to $75 \%$ (95\% confidence interval $59 \%$ to $91 \%$ ). This requires only a small reduction in the value of the upper limit of normal to $17 \cdot 5$.

If the two eyes without field loss are excluded from the early glaucoma group, then the sensitivity for detecting early field loss rises to $69 \%$ ( $95 \%$ confidence interval $51 \%$ to $87 \%$ ) for the original specificity of $96 \%$. Lowering the specificity to $90 \%$ produces a sensitivity for early field loss of $81 \%$ ( $95 \%$ confidence interval $66 \%$ to $96 \%)$.

Of particular interest was the distribution of eyes in the ocular hypertensive group, with $22 \%$ having an abnormal SBF score. The main drawback with this observation is whether these scores are repeatable, and this does require further study. However, abnormalities of visual function in ocular hypertensive patients have been reported in other studies assessing colour vision, ${ }^{78}$ contrast sensitivity, ${ }^{9}$ and with pattern electroretinography. ${ }^{10}$

Epidemiological studies suggest that approximately $10 \%$ of patients with ocular hypertension develop the typical field defects of glaucoma when followed up for 10 years. ${ }^{112}$ Our ocular hypertensive group, because of referral practices, may contain a greater proportion who will convert to glaucoma. This analysis may therefore offer additional information for use when attempting to assess which of these patients may progress to develop field loss, though a prospective follow-up study is required to evaluate this.

Recently it has been found that the use of a blue stimulus on a yellow background allows the isolation of short wavelength sensitive cones, and can detect glaucoma-like field defects that are undetectable with a standard white stimulus. ${ }^{8}$ Since our subjects wore the blue filter, this effectively produces a blue background, which should not stimulate the long and medium wavelength sensitive cones, thus creating similar conditions to those employed by Heron and colleagues, ${ }^{8}$ but by a different and simpler technique. More recent work, again using a blue stimulus on a yellow background, has confirmed its ability to detect early glaucomatous damage..$^{13}$ However, the authors also found that with dense defects (greater than $1.0 \mathrm{log}$ unit) blue/yellow perimetry showed no advantage over standard perimetry.

Isolated missed points on a suprathreshold screening programme are not uncommon in clinical practice, ${ }^{14}$ and they may cause considerable confusion. In the presence of an equivocal field defect on a white field an SBF score of 19 or less would indicate an $82 \%$ probability of normality. A score greater than this indicates a $90 \%$ probability of abnormality and would demand closer observation. However, this applies only to a typical hospital population of glaucoma suspects, where one-third of suspects would have early glaucoma.

In conclusion, although Logan and Anderson dismissed the use of a blue filter, ${ }^{5}$ we consider that its use, with our protocol, may aid the detection and confirmation of early loss of visual function in glaucoma suspects and merits further investigation.

We thank Mr T Arulampalam and Mr A S Rubasingham for kindly allowing us to study patients under their care.

1 de Monasterio FM. Asymmetry of on-and off-pathways of bluesensitive cones of the retina of macaques. Brain Res 1979; 166: $39-48$.

2 Quigley HA, Sanchez RM, Dunkelberger GR, L'Hernault NL, Baginski TA. Chronic glaucoma selectively damages large Baginski TA. Chronic glaucoma selectively damages large
optic nerve fibers. Invest Ophthalmol Vis $S$ ci 1987; 28:913-20.

3 Gündüz K, Arden GB, Perry S, Weinstein GW, Hitchings RA. Color vision defects in ocular hypertension and glaucoma. Quantification with a computer-driven color television system. Arch Ophthalmol 1988; 106: 929-35.

4 Yamazaki Y, Drance SM, Lakowski R, Schulzer M. Correlation between color vision and highest intraocular pressure in glaucoma patients. Am $\mathcal{F}$ Ophthalmol 1988; 106: 397-9.

5 Logan N, Anderson DR. Detecting early glaucomatous visua field changes with a blue stimulus. Am $\mathcal{f}$ Ophthalmol 1983 95: 432-4.

6 Heuer DK, Anderson DR, Feuer WJ, Gressel MG. The influence of refraction on automated perimetric threshold measurements. Ophthalmology 1987; 94: 1550-3.

7 Hamill TR, Post RB, Johnson CA, Keltner JL. Correlation of color vision deficits and observable changes in the optic disc in a population of ocular hypertensives. Arch Ophthalmol 1984 ; 102: 1637-9.

8 Heron G, Adams AJ, Husted R. Central visual fields for short wavelength sensitive pathways in glaucoma and ocula hypertension. Invest Ophthalmol Vis Sci 1988; 29: 64-72.

9 Ross JE, Bron AJ, Reeves BC, Emmerson PG. Detection of optic nerve damage in ocular hypertension. $\mathrm{Br} \mathcal{F}$ Ophthalmo 1985; 69: 897-903.

10 Weinstein GW, Arden GB, Hitchings RA, Ryan S, Calthorpe CM, Odom JV. The pattern electroretinogram (PERG) in ocular hypertension and glaucoma. Arch Ophthalmol 1988; 106: 923-8.

11 David R, Livingston DG, Luntz MH. Ocular hypertension - a long-term follow-up of treated and untreated patients. $\mathrm{Br} \mathcal{F}$ long-term follow-up of treated

12 Kitazawa Y, Horie T, Aoki S, Suzuki M, Nishioka K. Untreated ocular hypertension. A long-term prospective study. Arch Ophthalmol 1977; 95: 1180-4.

13 Hart Jr WM, Silverman SE, Trick GL, Nesher R, Gordon MO. Glaucomatous visual field damage. Luminance and colorcontrast sensitivities. Invest Ophthalmol Vis Sci 1990; 31:35967.

14 Henson DB, Dix SM, Oborne AC. Results of Friedmann Visual Field Analyser Mark II. Part 1. Results from a norma population. Br f Ophthalmol 1984; 68: 458-62. 